\title{
Management of a hospital-wide vancomycin-resistant Enterococcus faecium outbreak in a Dutch general hospital, 2014- 2017: successful control using a restrictive screening strategy
}

Veronica Weterings ${ }^{1 *} \mathbb{0}$, Anita van Oosten ${ }^{2}$, Ellen Nieuwkoop ${ }^{3}$, Jolande Nelson ${ }^{3}$, Andreas Voss ${ }^{4}$, Bas Wintermans ${ }^{2,7}$, Joris van Lieshout ${ }^{2}$, Jan Kluytmans ${ }^{1,5,6}$ and Jacobien Veenemans ${ }^{2,7}$

\begin{abstract}
Background: The emergence of vancomycin resistant enterococci poses a major problem in healthcare settings. Here we describe a hospital-wide outbreak of vancomycin-resistant Enterococcus faecium in a general hospital in The Netherlands in the period December 2014-February 2017. Due to late detection of the outbreak, a large cohort of approximately 25,000 (discharged) patients was classified as 'VRE suspected'. Hereupon a mitigated screening and isolation policy, as compared with the national guideline, was implemented to control the outbreak.

Methods: After the outbreak was identified, a screening policy consisting of a single rectal swab culture (with enrichment broth) to discontinue isolation and removing 'VRE suspected' label in the electronic patient files for readmitted VRE suspected patients, was implemented. In addition to the on admission screening, periodic hospital-wide point prevalence screening, measures to improve compliance with standard infection control precautions and enhanced environmental cleaning were implemented to control the outbreak.
\end{abstract}

Results: Between September 2014 and February 2017, 140 patients were identified to be colonised by vanA mediated vancomycin-resistant Enterococcus faecium (VREfm). Two of these patients developed bacteraemia. AFLP typing showed that the outbreak was caused by a single clone. Extensive environmental contamination was found in multiple wards. Within nine months after the detection of the outbreak no new VRE cases were detected.

Conclusion: We implemented a control strategy based on targeted screening and isolation in combination with implementation of general precautions and environmental cleaning. The strategy was less stringent than the Dutch national guideline for VRE control. This strategy successfully controlled the outbreak, while it was associated with a reduction in the number of isolation days and the number of cultures taken.

Keywords: VRE, Vancomycin-resistant enterococci, Resistance, Outbreak, Screening strategy, Isolation strategy

*Correspondence: vweterings@amphia.nl

${ }^{1}$ Department of Infection Control, Amphia Hospital, P.O. Box 90158, 4800 RK Breda, The Netherlands

Full list of author information is available at the end of the article

\section{Background}

In line with the increased global spread of multi-drug resistant microorganisms, the prevalence of vancomycin resistance among enterococci is rising. The European Antimicrobial Resistance Surveillance Network 
(EARS-Net) reported a significant increase of number of vancomycin-resistant Enterococcus faecium in invasive isolates from 2015 (10.5\%) to 2018 (17.3\%) in Europe [1]. In the Netherlands, however, the proportion of VRE in infection-related isolates remained stable and slightly higher than $1 \%$ over the past 5 years [2].

Despite this low and stable prevalence, there has been an increase in the number of VRE outbreaks. Whereas vancomycin-resistant enterococci (VRE) were detected only sporadically in the Netherlands before 2012, an increasing number of VRE outbreaks have required considerable time and resources to contain over the past 8 years [2]. In 2018 and 2019, VRE outbreaks were among the largest and the most frequently reported in the Netherlands (period 2018-June 2019: VRE 682 patients in 24 outbreaks versus MRSA 93 patients in 20 outbreaks) [3].

Due to the low virulence of VRE and its ability to survive on hospital environmental surfaces, VRE outbreaks have the potential to become substantial in size before they are detected by routine clinical cultures. Strategies to prevent VRE transmission include screening of contact patients and isolation precautions of (suspected) VRE carriers $[4,5]$. The Netherlands has a national control strategy of highly resistant micro-organisms including VRE [6]. During outbreaks, contact patients (those admitted to the same room or ward as the index patient) are pre-emptively isolated while awaiting test results to prevent further spread. In this context, there is ongoing discussion about the number of rectal swabs to be tested-with culture considered as gold standard-before a VRE suspected patient can be declared VRE negative. The negative predictive value of 1 negative rectal swab is considered insufficient, and the Dutch national guideline advocates taking 3-5 rectal cultures on separate days [6]. The combination of late outbreak detection and multiple cultures per contact-patient before VRE carriage can be excluded can result in very large numbers of patients to be isolated and screened, hence it is a substantial burden for hospital infection control departments, leading to significant laboratory costs, and exhaust hospital isolation facilities.

This paper describes an outbreak of VRE $f m$ in a general hospital in The Netherlands that was detected in December 2015, following an alert from a neighbouring hospital where VRE $f m$ carriage was detected in four recently transferred patients. A hospital-wide screening of all patients admitted for more than $48 \mathrm{~h}$ for VRE carriage indicated the spread of VRE among patients admitted to various departments at one of two hospital locations. The outbreak was successfully controlled slightly more than a year after its detection (February 2017).

This outbreak report provides an overview of the interventions that were implemented to control the outbreak, including a screening strategy that was more restrictive than the Dutch national guideline recommends.

\section{Methods \\ Design}

We retrospectively describe the interventions that were implemented to control an outbreak of VRE $f m$ that occurred between September 2014 and February 2017 in a 364-bed general hospital in a non-endemic setting in in the South West of the Netherlands.

\section{Setting}

The Admiraal De Ruyter Hospital (ADRZ) has approximately 25,000 admissions per year at two different locations (Goes and Vlissingen), and a catchment area of 248,000 inhabitants, and supplies $85-90 \%$ of the requested hospital care in this area. Tertiary care patients are referred to the surrounding academic centres. Most patients are admitted to an acute admission unit (AAU), consisting of six single rooms and eight multi-bed room with five beds each (total 46 beds), before being transferred to their specific wards (on average after $48 \mathrm{~h}$ ).

VRE bloodstream infections are very rare in the hospital: in the past 6 years no VRE bloodstream infections have been detected outside the outbreak period (Additional file 1: Materials S1). The antibiotic consumption rate in the hospital is relatively stable and low over the years: in 2016, the overall antibiotic use was 75.2 defined daily doses (DDD) per 100 patient days.

The internal infection control protocol for VRE was based on the Dutch national guideline for (targeted) screening and control measures [6]. This included contact isolation for VRE-positive patients; pre-emptive isolation and screening for patients with previous admission to foreign healthcare facilities and screening of roommates following an unexpected case of VRE. After discharge of a VRE positive patient, routine cleaning and disinfection of patient rooms with $250 \mathrm{ppm}$ chlorine was performed.

\section{Participants}

Participants were all patients admitted to the Admiraal De Ruyter Hospital (ADRZ) between September 1, 2014 and February 5, 2017. Patients were categorised into three groups according to their VRE status and potential risk of VRE carriage: (1) VRE carrier: VRE-positive patients; (2) VRE suspected patients: all patients with prior hospitalisation in the ADRZ hospital location Goes from September 1, 2014. In the beginning of the outbreak it was unclear whether the outbreak included neighbouring nursing homes or rehabilitation centres, therefore patients transferred from these institutions were also 
categorised as VRE suspected in the first phase of the outbreak.

Patients with no prior hospitalisation in the ADRZ hospital in the outbreak period, nursing home or rehabilitation centre were categorised as [3] low-risk patients.

\section{Interventions}

Upon detection of the outbreak in December 2015, an outbreak management team was initiated consisting of a clinical microbiologist, an infection control specialist, representatives of the hospital management and of the medical staff, the manager of housekeeping and logistics, and a member of the communication department.

The control measures can be divided into three phases, accompanied by the different screening and isolation policies. Table 1 summarizes the dates, isolation and screening policies per phase and risk group. In Table 2, an overview of the implemented control measures during the outbreak is shown.

VRE suspected patients were automatically labelled in the electronic patient system. The decision to preemptively isolate these patients, and the number of cultures required to remove the 'VRE suspected' label were adjusted in time over the course of the outbreak, and with the risk profile of the patients involved, as indicated in Table 1 and further specified in the results section below.

To detect unnoticed VRE transmission in the hospital, regular screening of all patients hospitalised for more than $48 \mathrm{~h}$, and those admitted to high risk units (ICU, dialysis) was implemented during the entire outbreak (Table 1).

\section{Audits, cleaning and education}

Cleaning tasks had to be performed by nurses or by dedicated cleaning personnel depending on the objects. During audits it became clear that the tasks had not been defined clearly and consequently some items were not always cleaned. As an intervention the tasks were specified in writing and subsequently the cleaning responsibilities were clearly defined. Also, damaged hospital equipment and furniture were repaired or replaced, and the cleaning frequency of sanitary facilities was increased. Audits on implementation of infection control measures and cleaning practises were performed, including adenosine triphosphate (ATP) measurements [7] performed by the infection prevention department. The ATP measurements were performed in patient rooms and common areas and to control cleaning after discharge (data not shown). All healthcare workers and cleaning personnel received mandatory training on standard infection control and cleaning policies. The number of alcohol-based hand rub (ABHR) dispensers was increased so ABHR was available at 'point of care' in all wards.

\section{Culturing and typing}

\section{Environmental sampling}

To assess the extent of environmental contamination in general (surveillance) and after cleaning and disinfection of the patient rooms, a range of high touch surfaces and (medical) equipment were sampled using $10 \mathrm{~cm} \times 10 \mathrm{~cm}$ sterile gauzes moistened with sterile saline [8]. See Additional file 1: Materials S2 for an overview of the most frequently sampled surfaces and equipment.

\section{Microbiology}

Rectal swabs or feces was collected by nursing staff using the eSwab medium (Copan, Murrieta, USA). In total, $100 \mu \mathrm{L}$ eSwab transport medium was transferred to a brain heart infusion broth containing $4 \mathrm{mg} / \mathrm{L}$ amoxicillin. After an overnight incubation at $35-37^{\circ} \mathrm{C}, 10 \mu \mathrm{L}$ of the broth was transferred to a Columbia colistin nalidixic acid-agar with 5\% Sheep Blood and vancomycin $(6 \mu \mathrm{g} /$ $\mathrm{mL}$ ) and grown overnight at $35-37^{\circ} \mathrm{C}$. For all suspected colonies growing on the selective media, species identification and susceptibility testing was performed using automated systems (Vitek MS and Vitek 2) (bioMérieux, Marcy l'Etoile, France) and E-test (bioMérieux, Marcy l'Etoile, France).

Resistance genes were detected using an in house $v a n A / v a n B$ duplex polymerase chain reaction (ElisabethTweeSteden Hospital, Tilburg, The Netherlands, see Additional file 1: Materials S3).

Multilocus sequence typing (MLST) [9] and Amplified fragment Length Polymorphism (AFLP) was used to type VRE isolates [10]. During the peak of the outbreak, typing was not performed when isolates carried a $\operatorname{VanA}$ gene and patients involved shared a clear epidemiological link to avoid unnecessary costs. Hence, further typing was performed for 62 isolates of VRE $f m$ positive patients (55 isolates of rectal screenings samples and 7 isolates of clinical samples) and three environmental isolates. An overview of the AFLP results is presented in Additional files 2 and 3.

\section{Results \\ Outbreak overview}

From September 2014 to February 2017, a total of 140 vancomycin-resistant Enterococcus faecium (VREfm) cases were identified. Figure 1 shows the epidemic curve of detection of new VRE $f m$-positive patients per week.

The VREfm strain found during this outbreak expressed a high level of resistance to vancomycin $(\mathrm{MIC}>256 \mathrm{mg} / \mathrm{L}$ ) and teicoplanin $(\mathrm{MIC}>32 \mathrm{mg} / \mathrm{L}$ ) and carried the vanA gene. VRE typing of one of the first isolates showed MLST ST117, designated AFLP type AT14181; subsequent AFLP analysis of 64 isolates in the 


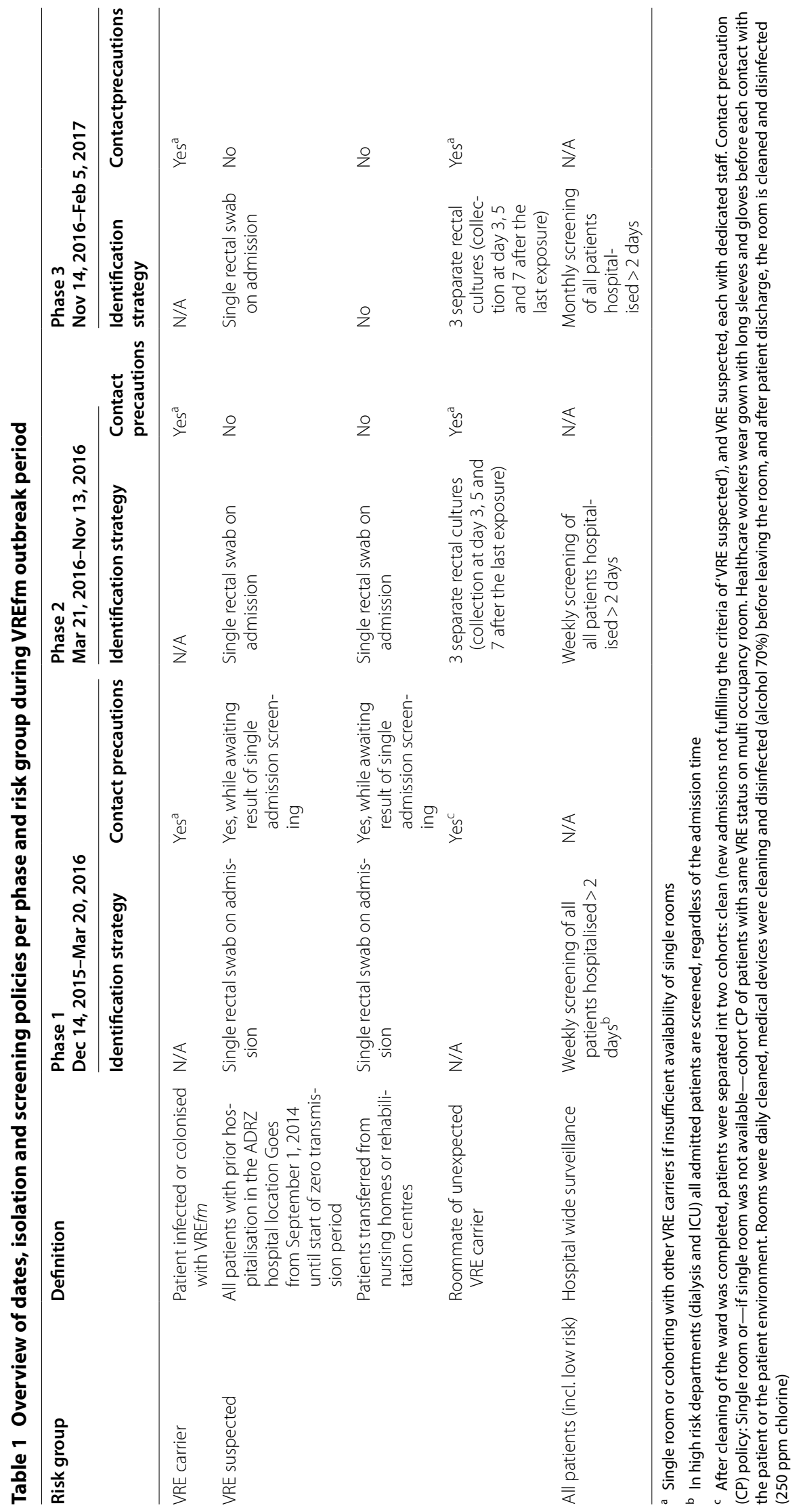


Table 2 Overview of the implemented control measures during VREfm outbreak period

\begin{tabular}{|c|c|c|}
\hline \multicolumn{3}{|c|}{ Overview of infection control measures during outbreak } \\
\hline 2015 & December (Phase I) & $\begin{array}{l}\text { Detection of the outbreak } \\
\text { Single hospital-wide screening limited to patients admitted for at least } 48 \mathrm{~h}\end{array}$ \\
\hline \multirow[t]{17}{*}{2016} & January & Initiation of Outbreak Management Team (OMT) \\
\hline & & $\begin{array}{l}\text { Reporting outbreak to the national Early warning and response meeting of Hospital-acquired Infections and } \\
\text { AntiMicrobial Resistance (SO-ZI/AMR) }\end{array}$ \\
\hline & & $\begin{array}{l}\text { Electronic labelling of VRE-positive patients (confirmed label) and patients with prior hospitalisation in het ADRZ } \\
\text { hospital in from September 1, 2014, or prior hospitalisation in a nursing home or rehabilitation centre ('VRE } \\
\text { suspected'label) }\end{array}$ \\
\hline & & Informing local hospital personnel and patients, and surrounding hospitals and nursing homes \\
\hline & & $\begin{array}{l}\text { Introducing screening for VRE carriage on admission for all patients with electronic 'VRE suspected' label and } \\
\text { patients from nursing homes or rehabilitation centres }\end{array}$ \\
\hline & & $\begin{array}{l}\text { Weekly hospital-wide VRE rectal screening limited to patients admitted for at least } 48 \mathrm{~h} \text {. In high risk departments } \\
\text { (dialysis and ICU) all admitted patients are screened, regardless of the admission time }\end{array}$ \\
\hline & & $\begin{array}{l}\text { Start of cleaning and disinfection ( } 250 \text { ppm chlorine) of the entire hospital: wards are cleaned one by one, } \\
\text { whereby patients are temporarily transferred to other (not yet cleaned) parts of the same or other wards }\end{array}$ \\
\hline & & Introduction of disinfectant wipes for contact surfaces in patient rooms and general areas \\
\hline & & $\begin{array}{l}\text { Start of mandatory plenary training sessions for all healthcare workers on general precautions and cleaning } \\
\text { issues }\end{array}$ \\
\hline & & Clear division of cleaning tasks for healthcare workers and cleaning personnel \\
\hline & February & $\begin{array}{l}\text { After cleaning of the ward: release rooms previously occupied by VRE positive patients after cleaning and disin- } \\
\text { fection based on environmental cultures }\end{array}$ \\
\hline & March (Phase II) & Audits of adherence to infection control and cleaning protocols by infection control department \\
\hline & & $\begin{array}{l}\text { Implementing screening and isolation protocol for 'high risk' patients (direct contacts of VRE carriers, mostly } \\
\text { roommates) }\end{array}$ \\
\hline & & $\begin{array}{l}\text { Reintroducing of cleaning and disinfection ( } 250 \mathrm{ppm} \text { chlorine) of the entire hospital: departments are cleaned } \\
\text { one by one, whereby patients are temporarily transferred to other (not yet cleaned) departments }\end{array}$ \\
\hline & & Intensifying communication to healthcare workers and managers \\
\hline & November (Phase III) & $\begin{array}{l}\text { Screening for VRE carriage upon admission limited to only patients with prior hospitalisation in het ADRZ hospi- } \\
\text { tal in period December 1, 2015-November } 14,2016\end{array}$ \\
\hline & & $\begin{array}{l}\text { Monthly hospital-wide VRE rectal screening limited to patients admitted for at least } 48 \mathrm{~h} \text {. In high risk depart- } \\
\text { ments (dialysis and ICU) all admitted patients are screened, regardless of the admission time }\end{array}$ \\
\hline \multirow[t]{2}{*}{2017} & February (End of the outbreak) & Removing all outbreak related 'VRE suspected' labels in the electronic patient system \\
\hline & & Start hospital-wide VRE rectal screening limited to patients admitted for at least 7 days \\
\hline
\end{tabular}

course of the outbreak period revealed a single AFLP clone (Additional file 2).

The outbreak was detected in December 2015, where VRE $f m$ carriage was found in four patients who had been transferred from location Goes. On December 14, 2015, following the alert from a neighbouring academic hospital, all patients admitted for more than $48 \mathrm{~h}$ were screened for VRE carriage: of the 158 patients screened, 13 patients (8\%) were carriers of VRE $f m$. The VRE $f m$ positive patients were all admitted to various departments at location Goes (none at location Vlissingen), including the Intensive Care Unit, indicating that VRE $f m$ had spread throughout the entire location (with the exception of the AAU, and the children's and maternity ward).

Retrospectively, the start of the outbreak was set at September 1, 2014, as on this date the first VREfm was detected in a clinical specimen of a patient admitted to the surgical ward at location Goes. In the months following September 2014, VRE $f m$ strains had been isolated in clinical materials from five patients during their admission to location Goes (Fig. 1). These had been at the time considered to be incidental findings without a clear epidemiological link. AFLP typing in December 2015 showed that these isolated belonged to a single AFLP clone (Additional file 2).

In the first phase out the outbreak from January 2016, VRE suspected patients were isolated with contact precautions (preferably in single rooms) upon (re)admission. Isolation measures were discontinued and the 'VRE suspected' label was removed based on a single negative rectal culture. Due to the large number of VRE suspected patients and insufficient availability of single rooms, separate cohorts were formed for VRE positive and VRE suspected patients, each with dedicated rooms, staff and (medical) equipment. Two extra five-bed-rooms were opened on the AAU to accommodate these cohorts by 


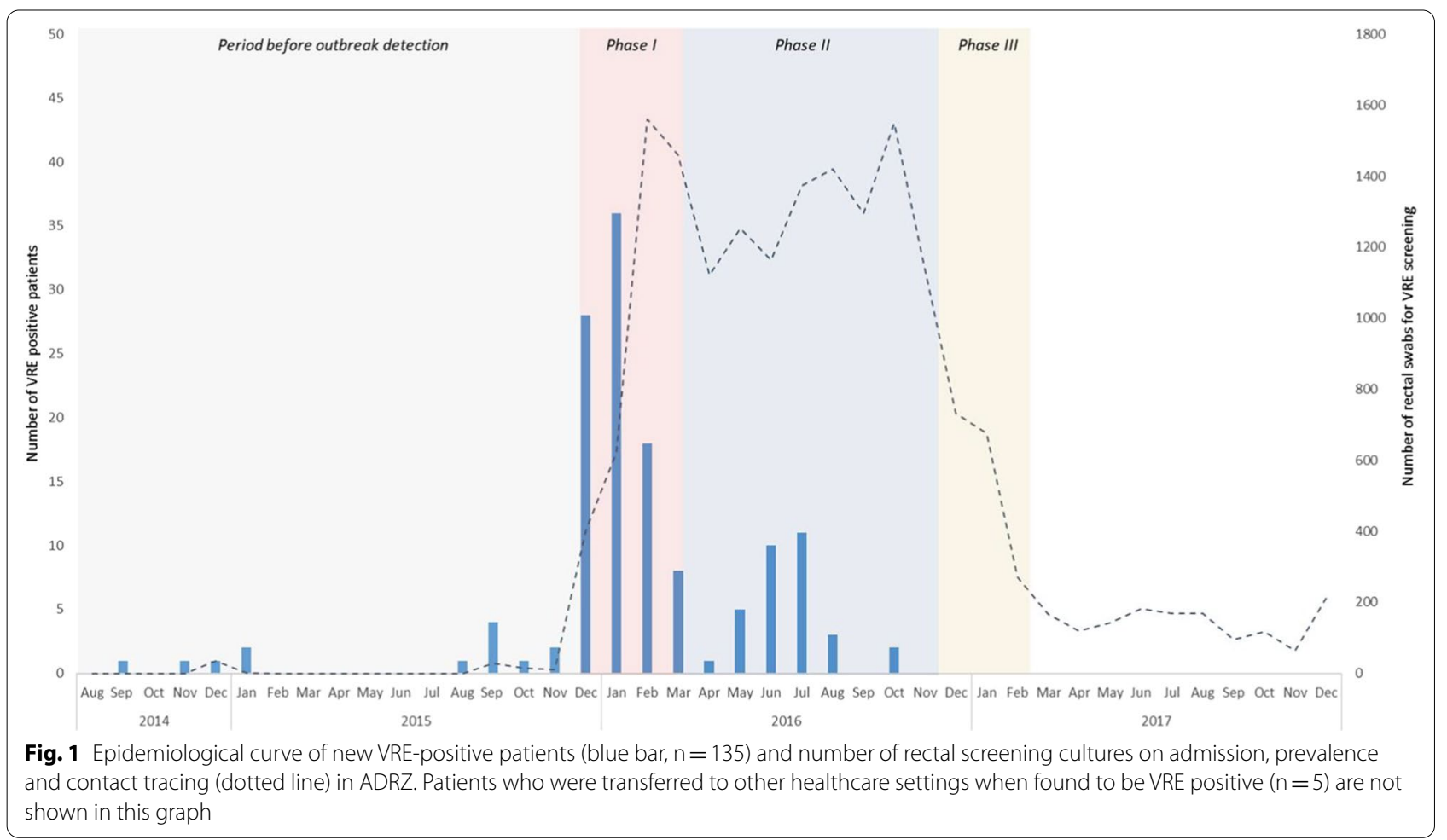

mid-January 2016. On the other wards, VRE suspected or positive patients were placed in a single room or cohorted with other (suspected) VRE carriers if there was insufficient availability of single rooms.

Screening of VRE suspected patients upon admission between January and March 2016 (phase I), showed that only 2 of 647 patients $(0.31 \%)$ were positive for VRE $f m$. None of these patients were residing in a nursing home or rehabilitation facility. Given the low VRE prevalence in the cohort of patients admitted between September 2014 and December 2015 and the absence of VRE carriage among patients transferred from other institutions, patients from this cohort were labelled 'medium risk' and no longer pre-emptively isolated upon admission starting from March 21, 2016 (phase II). Screening of this cohort by performing a single rectal culture upon admission remained unchanged.

In this second phase of the outbreak, patients who had been in contact with a VREfm-positive patient (roommates of VRE carriers) were labelled 'high risk', isolated and screened using 3 separate rectum cultures on day 3, 5 and 7 after last exposure (Table 1).

After an initial decline in the number of new VREfm findings in February and March, 2016-there was a second peak in the number of VRE $f m$ positive patients in May-June, 2016. On-site audits performed during phase II showed shortcomings in infection control preconditions on several wards in the hospital: wards were cluttered, the separation of dirty and clean areas was not clear, and the replacement of damaged hospital equipment and furniture had not been implemented. The division of labour with regards to cleaning responsibilities between cleaning personnel and healthcare workers was further emphasized in this phase, and environmental sampling increased in frequency (see below).

As of November 2016, there were no new VRE $f m$ findings in the preceding three months and therefore screening for VRE carriage on admission was limited to only patients with prior hospitalisation in het ADRZ hospital in the period December, 2015-November, 2016 (phase III). Furthermore, the frequency of hospital-wide screening of patients with $>48 \mathrm{~h}$ length of stay was from then on performed monthly instead of weekly.

\section{Control of the outbreak}

No further cases occurred over a three months period and control measures were terminated in February, 2017. Admission and prevalence surveillance cultures were discontinued and all outbreak related 'VRE suspected' labels in the electronic patient system were removed. Furthermore, a hospital-wide VRE rectal screening limited to patients admitted for at least 7 days, was implemented as a standard surveillance form that moment on. 


\section{Environmental cultures}

In January 2016, environmental cultures were obtained throughout the hospital to assess the extent of environmental contamination. The cultures showed extensive VRE contamination on the surgical, internal, pulmonary and neurology wards (43/80 samples VRE positive; 53.7\%). Environmental samples of the AUU, ICU and dialysis department were VRE negative (0/60 samples). (Fig. 2a).

In June 2016, environmental screening was repeated on multiple wards ( $n=130$ samples), and again extensive VRE contamination was found in the surgical ward (19/20 samples, 95.0\%) and to a lesser extent on the cardiology ward (4/20 samples; 20.0\%). Consequently, stepwise cleaning and disinfection (250 ppm chlorine) of these wards was performed. After cleaning these wards were closed pending VRE negative environmental results. Following a peak in VRE transmission, environmental surveillance was continued and intensified: from June 2016, rooms previously occupied by VRE-positive patients were only released after cleaning and negative environmental cultures. Ten percent (74/713 culture) of the room surfaces remained VRE $f m$ positive after terminal disinfection (Fig. 2b). In some cases, VREfm was still detected after two rounds of terminal disinfection on e.g. patient bed, infusion pole and pull-up bar.

\section{Infections during the outbreak period}

Eight $(5.7 \%)$ patients developed a VREfm infection, of whom two (1.4\%) had bacteraemia. Two patients, with extensive co-morbidities, died shortly after detection. One patient, also with extensive co-morbidities (including renal failure, haemodialysis and vascular disease) developed a severe osteomyelitis following a surgical procedure, which eventually led to amputation of her left hand.

\section{Discussion}

Here we describe the successful control of a VREfm outbreak in a hospital using a more restrictive screening and isolation policy than recommended in the national guidelines [6]. With this approach, within nine months after the detection of the outbreak, no new VREfm cases were detected and after twelve months the outbreak was considered fully controlled. In addition to the targeted screening and isolation there was an intensive focus on optimisation of environmental cleaning procedures.

In general, there is no consensus on the optimal VRE screening, isolation and surveillance protocol, reflected by the variation in infection control approaches within and between countries [11-13]. The number of rectal cultures required to consider a known carrier or contact patient VRE-negative, is unclear. Studies show that the sensitivity of a single rectal swab is low, ranging from 42.5 to $79 \%$ [14-17], and this increases when taking multiple swabs: Pearman et al. showed that on average four rectal swabs, collected on separate days, were needed to detect $95 \%$ of carriers compared to $56 \%$ with one rectal swab [15]. Explanations for the increase in sensitivity when taking multiple rectal swabs include a fluctuation in faecal excretion of VRE, and/or the presence of an intestinal transit time after VRE is transmitted (time between transmission event and detectable VRE levels in the faeces). It should be noted that in most of these studies the rectal swabs were inoculated directly on selective media. Addition of a broth enrichment step (as done in our study) increases the yield of a rectal swab culture substantially [18]. Lastly, sensitivity may depend on the load of VRE in faeces [17]; a high VRE load in faeces also results in a higher sensitivity of a rectal swab, whereby patients with lower faecal loads probably contribute less to transmission.

In 2015, a Dutch guideline was published which recommends taking 3-5 rectal cultures on separate days to reliably exclude carriage in a suspected VRE carrier [6]. This guideline makes no specific recommendation about the timing of the 3-5 separate rectal culture collection, apart from recommending that the last culture is ideally performed at least seven days after the last exposure. During our outbreak, the screening policy consisting of a single rectal swab culture (with enrichment broth) upon readmission for excluding VRE carriage in the majority of VRE suspected patients in the first phase of the outbreak, and a maximum of three culture in the second phase for 'high risk' VRE suspected patients.

In this outbreak, there was a long period of time between the first clinical VRE finding (September 2014) and the detection of the outbreak (December 2015), resulting in a large cohort of approximately 25,000 discharged and potentially exposed patients, hence classified as a 'VRE suspected'. Readmission of these patients occurred frequently and it was estimated, based on historical data, that approximately 4700 admissions/year would be categorised as 'VRE suspected'. According to the guideline this would result in 75,000-125,000 cultures to detect all carriers (by screening all discharged patients). This would have required considerable administrative effort and laboratory costs. In case of limiting the screening to those who were re-admitted (appr. 4700/ year), isolating all 'VRE suspected' patients in a single room upon admission pending at least 3 rectal screening cultures on separate days would place a large burden on hospital room capacity, and still require a very large number of cultures.

With the assumption that the sensitivity of a single swab is substantially higher when performed $>7$ days 
a

Environmental cultures - surveillance

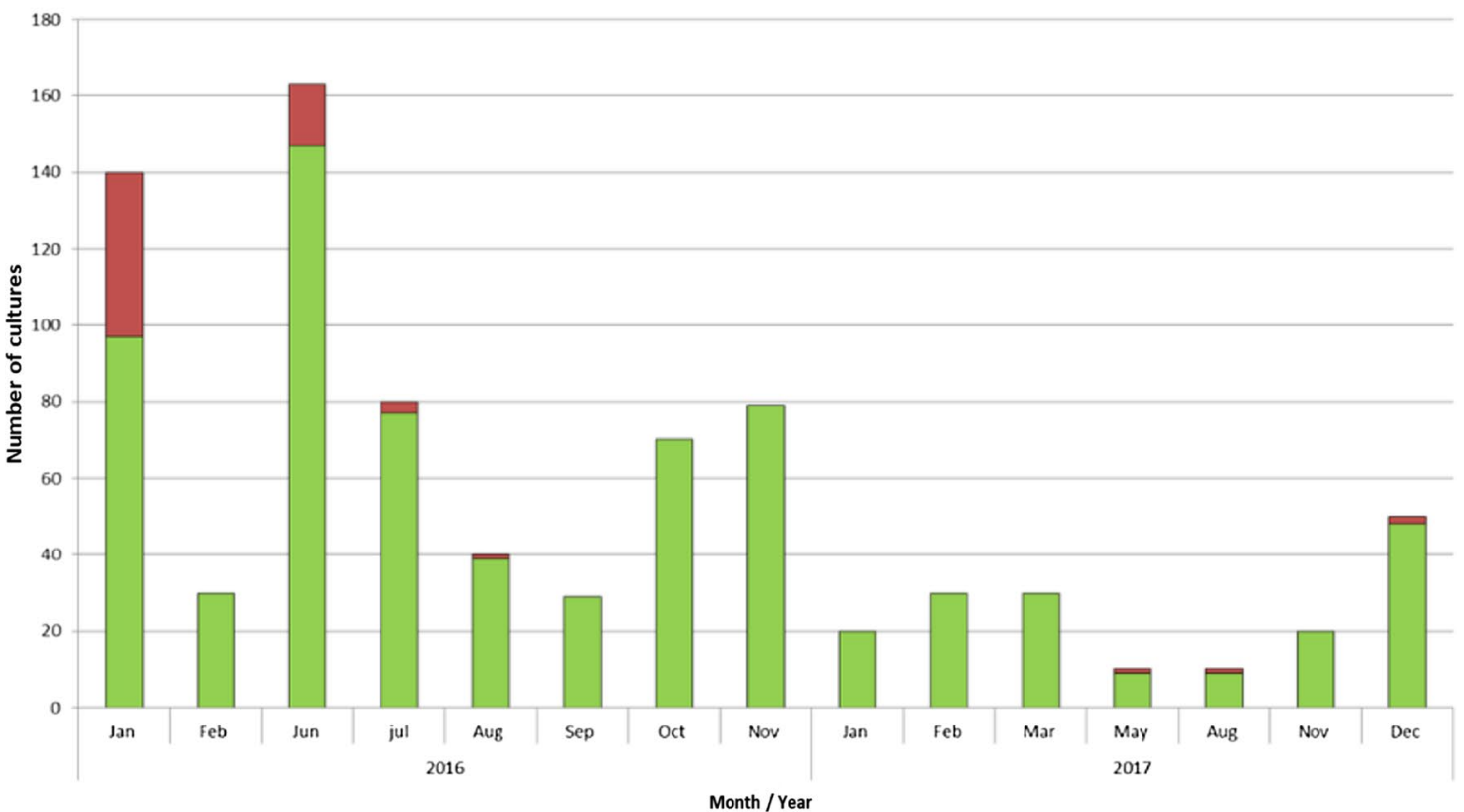

b

Environmental cultures - after terminal desinfection

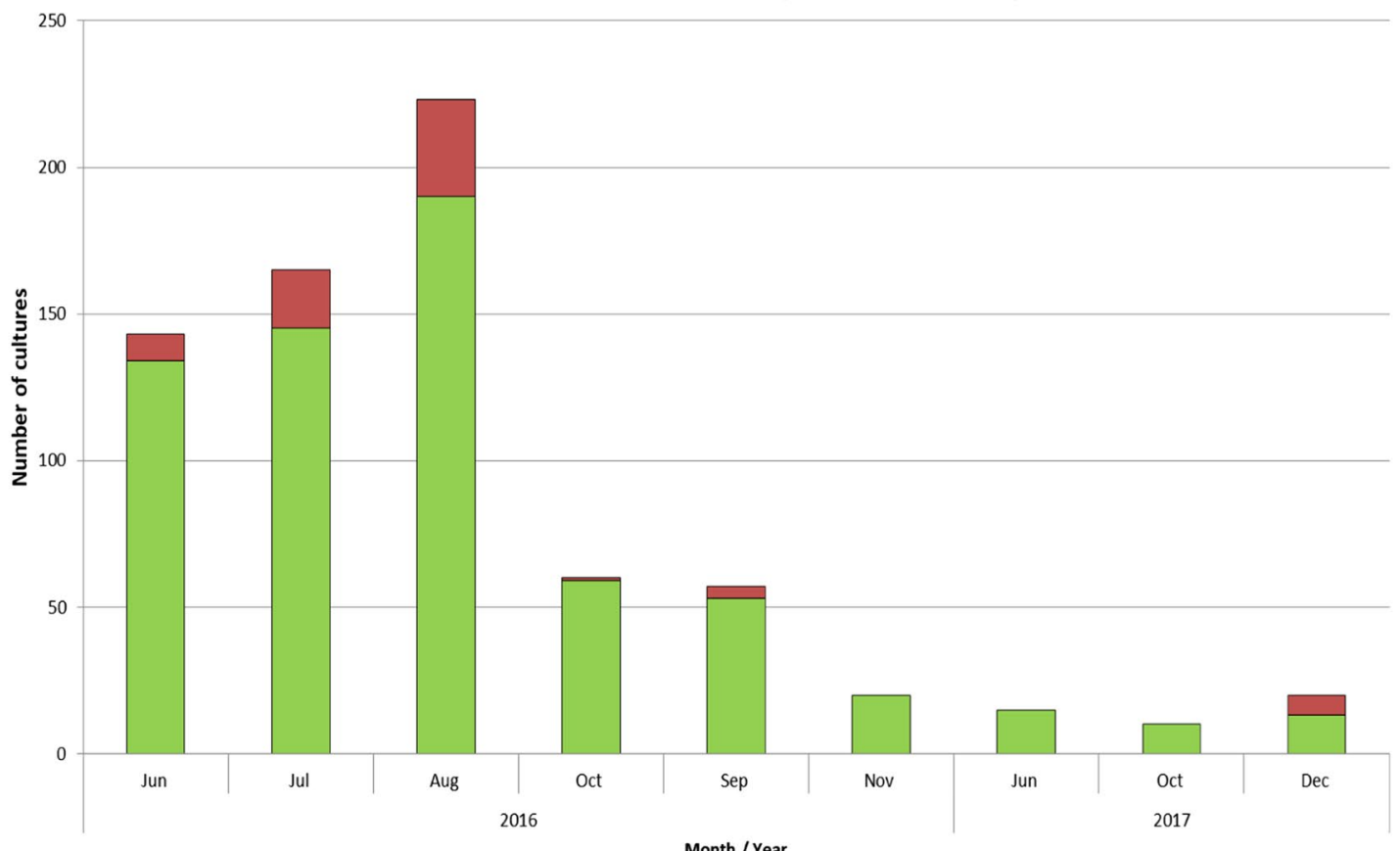

Fig. 2 Environmental cultures of the patient rooms, a range of high touch surfaces and (medical) equipment's at random times (surveillance) (a) and after disinfection (b). Red bar:VREfm positive cultures; green bar: VRE negative cultures

after the last exposure, and because a substantial proportion of exposed patients was expected to have lost VRE carriage within 6 months [19], we decided to perform screening for VRE by taking a single swab, and limit screening to patients who were re-admitted to the hospital. By ending pre-emptive contact precautions after a single VRE-negative rectal swab, most patients were isolated only the first two days of admission. With this strategy, that was less stringent than the Dutch national recommendations for VRE control, a total of 19,677 
rectal swabs (of 9279 patients) were collected during the entire outbreak (admission-, prevalence- and contact surveillance cultures together), thereby significantly cutting administrative effort, time of isolation and laboratory costs.

Though it can be argued that we have not detected all VRE $f m$ carriers (and underestimated the size of the outbreak) due to suboptimal sensitivity of our screening policy, our experience shows that VRE transmission from undetected carriers was, even if present, insufficient to caused sustained transmission. Whether this strategy would have been effective in settings with higher complexity of care (where patients probably have longer duration of carriage, longer admissions, and a higher transmission potential and infection risk) is unclear. In such settings, even a small loss in sensitivity may lead to ongoing transmission. In a recent paper, Frakking et al. describe a successful control of a large VRE outbreak $(\mathrm{n}=242$ patients) in a Dutch teaching hospital and tertiary referral centre [4]. The outbreak lasted 18 months and was eventually controlled after major efforts, including twice-weekly screening of all admitted patients, environmental decontamination with hydrogen peroxide vapour, strict isolation precautions (in isolation rooms with anteroom), a VRE quarantine ward and abandoning the use of ciprofloxacin prophylaxis during neutropenic fever. The authors advocate a screening policy with at least 4-5 rectal swabs before excluding VRE carriage.

It should be noted that in our study, for the majority of the cohort of suspected VRE patients the last exposure was $>7$ days ago (for many patients even several months), which (in combination with intensive focus on general precautions and environmental cleaning) may have been an important reason for the success of this strategy. In addition, the prevalence among re-admitted patients was $<1 \%$, indicating a low background risk of undetected introductions of VRE in the hospital.

As described we are careful to generalize our findings to other settings. Our results suggest, however, that the current general Dutch recommendations to take 3-5 cultures to exclude VRE carriage in all exposed patients may be reconsidered for centres with lower complexity of care, especially when the last exposure was $>7$ days ago. This lowers the costs and limits the duration of pre-emptive isolation.

\section{Conclusion}

To conclude, we describe a large VRE outbreak in a general hospital in The Netherlands, that was successfully controlled, while substantially reducing the number of cultures to be taken and the number of isolation days, and thereby cutting laboratory costs.

\section{Supplementary information}

The online version contains supplementary material available at https://doi. org/10.1186/s13756-021-00906-x.

\section{Additional file 1. Additional files.}

Additional file 2. Dendrogram based on Amplified fragment length polymorphism (AFLP) of 65 VRE strains identified during the outbreak. E. faecium (CDC NY2) and E. faecalis (ATCC 29212) were included in the analysis as reference strains. The cut-off value for identical strains was $90 \%$ relative similarity.

Additional file 3. Dendrogram based on Amplified fragment length polymorphism (AFLP) of 12 VRE strains identified between 2016 and 2020 in ADRZ - NOT belonging to the outbreak cluster. E. faecium (CDC NY2) and E. faecalis (ATCC 29212) were included in the analysis as reference strains. The cut-off value for identical strains was $90 \%$ relative similarity.

\section{Abbreviations}

AAU: Acute admission unit; ADRZ: Admiraal De Ruyter Hospital (ADRZ); AFLP: Amplified fragment length polymorphism; ICU: Intensive care unit; MLST: Multilocus sequence typing; MRSA: Methicillin-resistant Staphylococcus aureus; VRE: Vancomycin-resistant enterococci; VREfm: Vancomycin-resistant Enterococcus faecium.

\section{Acknowledgements}

We would like to acknowledge the healthcare workers, colleagues of the department of Infection control and Laboratory for Microbiology of the Admiraal De Ruyter Hospital-who contributed to the investigation and the control of this outbreak. Furthermore, the authors thank the department of Medical Microbiology and Infection Control of Amsterdam UMC for the AFLP analysis and dendrogram construction.

\section{Authors' contributions}

$A \vee O, E N, J N, B W, J V L, J K$ and JV performed the investigation and management of the outbreak. VW and JV did the data analysis. VW wrote the first draft of the manuscript. AvO, EN, AV, JN, BW, JVL, JK and JV reviewed, provided critical feedback and contributed to subsequent draft. All authors read and approved the final manuscript.

Funding

Not applicable.

\section{Availability of data and materials}

The datasets used and/or analysed during the current study are available from the corresponding author on reasonable request.

\section{Ethics approval and consent to participate}

The data of patients used in this study were part of routine clinical practices in the ADRZ hospital and their anonymous use is beyond the scope of the Medical Research Involving Human Subjects Act.

\section{Consent for publication}

Not applicable.

\section{Competing interests}

The authors declare that they have no competing interests.

\section{Author details}

${ }_{1}^{1}$ Department of Infection Control, Amphia Hospital, P.O. Box 90158, 4800 RK Breda, The Netherlands. ${ }^{2}$ Department of Infection Control, Admiraal De Ruyter Hospital, P.O. Box 15, 4460 AA Goes, The Netherlands. ${ }^{3}$ Department of Infection Control, Elisabeth-TweeSteden Hospital, P.O. Box 90151, 5000

LC Tilburg, The Netherlands. ${ }^{4}$ Department of Medical Microbiology, Radboud University Medical Centre, P.O. Box 9101, 6500 HB Nijmegen, The Netherlands. ${ }^{5}$ Microvida Laboratory for Microbiology, Amphia Hospital, P.O. Box 90158 , 4800 RK Breda, The Netherlands. ${ }^{6}$ Julius Center for Health Sciences and Primary Care, UMC Utrecht, Utrecht University, P.O. Box 85500, 3508 GA Utrecht, the Netherlands. ${ }^{7}$ Laboratory for Microbiology, Admiraal De Ruyter Hospital, P.O. Box 15, 4460 AA Goes, The Netherlands. 
Received: 22 August 2020 Accepted: 3 February 2021

Published online: 18 February 2021

\section{References}

1. European Centre for Disease Prevention and Control. Surveillance of antimicrobial resistance in Europe 2018. Stockholm: ECDC; 2019.

2. Nethmap 2019. Consumption of antimicrobial agents and antimicrobial resistance among medically important bacteria in the Netherlands in 2018. https://www.rivm.nl/documenten/nethmap-2019.

3. Overzicht meldingen aan het signaleringsoverleg zorginfecties en antimicrobiële resistentie (SO-ZI/AMR) ZI/AMR) Tabel. Overzicht van meldingen aan het SO-ZI/AMR die werden gemeld en/of afgerond in 2018 en 2019 en eerder gepubliceerd in de tabellen van het Wekelijks overzicht. 2019;1-5. https://www.nvmm.nl/media/2910/2018-2019-totaal-overz icht-van-meldingen-na-2019-07.pdf.

4. Frakking FNJ, Bril WS, Sinnige JC, Klooster JEV, de Jong BAW, van Hannen EJ, et al. Recommendations for the successful control of a large outbreak of vancomycin-resistant Enterococcus faecium in a non-endemic hospital setting. J Hosp Infect. 2018;100(4):e216-25.

5. Werkgroep Infectiepreventie. 2012. WIP-richtlijn BRMO (Bijzonder Resistente Micro-Organismen). http://www.rivm.nl/Documenten_en_publi caties/Professioneel_Praktisch/Richtlijnen/Infectieziekten/WIP_Richtlijne n/Actuele_WIP_Richtlijnen/Ziekenhuizen/WIP_richtlijn_BRMO_Bijzo nder_Resistente_Micro_Organismen_ZKH.

6. Sinnige JC, Willems RJL, Ruijs GJHM, Mascini E, Arends JP, Troelstra A. NVMM Guideline HRMO VRE. 2015. http://www.nvmm.nl/media /1049/2015_hrmo_vre.pdf.

7. Van Arkel A, Willemsen I, Kilsdonk-Bode L, Vlamings-Wagenaars S, van Oudheusden A, De Waegemaeker P, et al. ATP measurement as an objective method to measure environmental contamination in 9 hospitals in the Dutch/Belgian border area. Antimicrob Resist Infect Control. 2020;9(1):77. https://doi.org/10.1186/s13756-020-00730-9.

8. Weterings V, Zhou K, Rossen JW, van Stenis D, Thewessen E, Kluytmans $J$, et al. An outbreak of colistin-resistant Klebsiella pneumoniae carbapenemase-producing Klebsiella pneumoniae in the Netherlands (July to December 2013), with inter-institutional spread. Eur J Clin Microbiol Infect Dis. 2015;34(8):1647-55.

9. De Been M, Pinholt M, Top J, Bletz S, Mellmann A, Van Schaik W, et al. Core genome multilocus sequence typing scheme for high-resolution typing of enterococcus faecium. J Clin Microbiol. 2015:53(12):3788-97.
10. Savelkoul PHM, Aarts HJM, De Haas J, Dijkshoorn L, Duim B, Otsen M, et al. Amplified-fragment length polymorphism analysis: the state of an art. J Clin Microbiol. 1999:37(10):3083-91.

11. Isenman H, Michaels J, Fisher D. Global variances in infection control practices for vancomycin resistant Enterococcus - results of an electronic survey. Antimicrob Resist Infect Control. 2016;5:41.

12. Martischang R, Buetti N, Balmelli C, Saam M, Widmer A, Harbarth S. Nation-wide survey of screening practices to detect carriers of multi-drug resistant organisms upon admission to Swiss healthcare institutions. Antimicrob Resist Infect Control. 2019;8(1):1-4.

13. Buetti N, Wassilew N, Rion V, Senn L, Gardiol C, Widmer A, et al. Emergence of vancomycin-resistant enterococci in Switzerland: a nation-wide survey. Antimicrob Resist Infect Control. 2019;8:16.

14. Weinstein JW, Tallapragada S, Farrel P, Dembry LM. Comparison of rectal and perirectal swabs for detection of colonization with vancomycinresistant enterococci. J Clin Microbiol. 1996;34(1):210-2.

15. Pearman J, Perry P, Kosaras F, Douglas C, Lee R, Peterson A, et al. Screening and electronic labelling of ward contacts of vancomycin-resistant Enterococcus faecium vanB carriers during a single-strain hospital outbreak and after discharge from hospital. Commun Dis Intell. 2003;1 (27 Suppl):S97-102.

16. Kaki R, Yu Y, O'Neill C, Lee C, Mertz D. Vancomycin-resistant enterococcus (VRE) transmission and risk factors in contacts of VRE carriers. Infect Control Hosp Epidemiol. 2014;35(7):876-9.

17. D'Agata EMC, Gautam S, Green WK, Tang Y-W. High rate of false-negative results of the rectal swab culture method in detection of gastrointestinal colonization with vancomycin-resistant enterococci. Clin Infect Dis. 2002;34(2):167-72.

18. leven M, Vercauteren E, Descheemaeker P, Van Laer F, Goossens H. Comparison of direct plating and broth enrichment culture for the detection of intestinal colonization by glycopeptide-resistant enterococci among hospitalized patients. J Clin Microbiol. 1999;37(5):1436-40.

19. Sohn KM, Peck KR, Joo E-J, Ha YE, Kang C-I, Chung DR, et al. Duration of colonization and risk factors for prolonged carriage of vancomycinresistant enterococci after discharge from the hospital. Int J Infect Dis. 2013;17(4):e240-6.

\section{Publisher's Note}

Springer Nature remains neutral with regard to jurisdictional claims in published maps and institutional affiliations.
Ready to submit your research? Choose BMC and benefit from:

- fast, convenient online submission

- thorough peer review by experienced researchers in your field

- rapid publication on acceptance

- support for research data, including large and complex data types

- gold Open Access which fosters wider collaboration and increased citations

- maximum visibility for your research: over $100 \mathrm{M}$ website views per year

At BMC, research is always in progress.

Learn more biomedcentral.com/submissions 